\title{
Evaluation of Efficacy of Standard Treatment of Non-Purulent Cellulitis
}

\author{
Abdalla Abd Al-Hamid Sallam', Mohammed Rashed Abd Al-Hamid², Mohammed Abd Al-Aale Yusuf \\ Ali Nafie', Abdalla Mohammed Al-Hady Abd Al-Aziz ${ }^{1}$ \\ ${ }^{1}$ Department of General Surgery, ${ }^{2}$ Department of Clinical Pathology, Faculty of Medicine, Al-Azhar \\ University, Cairo, Egypt
}

*Corresponding author: Abdalla Mohammed Al-Hady Abd Al-Aziz, Mobile: (+20) 1020279742, E-Mail:

abdallahabdalaziz35@gmail.com

\begin{abstract}
Backgound: Cellulitis is commonly used to indicate a nonnecrotizing inflammation of the skin and subcutaneous tissues, a process usually related to acute infection that does not involve the fascia or muscles. Cellulitis is characterized by localized pain, swelling, tenderness, erythema, and warmth, cellulitis has been classically considered to be an infection without formation of abscess (nonpurulent), purulent drainage, or ulceration.

Objective: The aim of the current work was to evaluate the efficacy of standard treatment for cases of cellulitis and identify other treatment options if needed in local community.

Patients and methods: This prospective study was conducted at Department of General Surgery, Al-Azhar university hospitals (Al-Hussin \& Said Galal hospitals). This study carried out on 100 consecutive patients with non-purulent cellulitis from 23/2/2017 to 1/5/2018 to allow a minimum follow-up period of at least 4 months for the last case follow upon. Clinical response to dicloxacillin, amoxicillin, and cephalexin was evaluated.
\end{abstract}

Results: Age, sex, presentation, sit of non purulent cellulitis and result were recorded. Cellulitis was found to be more common in geriatric patients and slightly more in females. Most patients had lower limb cellulitis, and only little patients had an additional sit as orbit, hand and genitalia. 83 cases have good clinical response and 17 cases have poor clinical response within 7 days to dicloxacillin, amoxicillin, and cephalexin.

Conclusion: Dicloxacillin, amoxicillin, or cephalexin are effective in most cases of cellulitis with gram positive organisms as Streptococcal and staphylococcal species.

Keywords: Non-Purulent Cellulitis, dicloxacillin, amoxicillin, cephalexin

\section{INTRODUCTION}

The term cellulitis is commonly used to indicate a nonnecrotizing inflammation of the skin and subcutaneous tissues, usually from acute infection (see the image below). Cellulitis usually follows a breach in the skin, although a portal of entry may not be obvious; the breach may involve microscopic skin changes or invasive qualities of certain bacteria. Signs and symptoms: nonpurulent cellulitis is associated with the 4 cardinal signs of infection, as follows: Erythema, Pain, Swelling and warmth. Physical examination findings that suggest the most likely pathogen include the following: Skin infection without underlying drainage, penetrating trauma, eschar, or abscess is most likely caused by streptococci; Staphylococcus aureus, often community-acquired MRSA, is the most likely pathogen when these factors are present ${ }^{(\mathbf{1}, \mathbf{2})}$.

Violaceous color and bullae suggest more serious or systemic infection with organisms such as Vibrio vulnificus or Streptococcus pneumoniae. The following findings suggest severe infection: Malaise, chills, fever, toxicity, Lymphangitic spread (red lines streaking away from the area of infection), Circumferential cellulitis and Pain disproportionate to examination findings. Indications for emergent surgical evaluation are as follows: Violaceous bullae, Cutaneous hemorrhage, Skin sloughing, Skin anesthesia, Rapid progression, Gas in the tissue and Hypotension ${ }^{(3)}$.

Generally, no workup is required in uncomplicated cases of cellulitis that meet the following criteria: Limited area of involvement, Minimal pain, no systemic signs of illness (eg, fever, altered mental status, tachypnea, tachycardia, hypotension) and no risk factors for serious illness (eg, extremes of age, general debility, immunocompromise). The Infectious Disease Society of America (IDSA) recommends the following blood tests for patients with softtissue infection who have signs and symptoms of systemic toxicity: Blood cultures, CBC with differential, And levels of creatinine, bicarbonate, creatine phosphokinase, and C-reactive protein $(\mathrm{CRP})^{(4)}$. 
Blood cultures should also be done in the following circumstances: Moderate to severe disease $^{(\mathbf{3})}$ (eg, cellulitis complicating lymphedema) (5). Cellulitis of specific anatomic sites (eg, facial and especially ocular areas), patients with a history of contact with potentially contaminated water ${ }^{(6)}$, patients with malignancy who are receiving chemotherapy, neutropenia or severe cell-mediated immunodeficiency and animal bites. Other tests to consider are as follows: Mycologic investigations are advisable if recurrent episodes of cellulitis are suspected to be secondary to tinea pedis or onychomycosis. creatinine levels help assess baseline renal function and guide antimicrobial dosing ${ }^{(3)}$.

Imaging studies: Ultrasonography may play a role in the detection of occult abscess and direction of care, ultrasonographic-guided aspiration of pus can shorten hospital stay and fever duration in children with cellulitis, if necrotizing fasciitis is a concern, CT imaging is typically used in stable patients; MRI can be performed, but MRI typically takes much longer than CT scanning and strong clinical suspicion of necrotizing fasciitis should prompt surgical consultation without delay for imaging ${ }^{(7)}$.

Inpatient admission is recmmended in patients with hypotension and/or the following laboratory findings: Elevated creatinine level, elevated creatine phosphokinase level (2-3 times the upper limit of normal), CRP level $>13 \mathrm{mg} / \mathrm{L}$ (123.8 $\mathrm{mmol} / \mathrm{L})$, low serum bicarbonate level and CBC showed marked shift to left ${ }^{(3)}$.

Treatment of cellulitis is as follows: Antibiotic regimens are effective in more than $90 \%$ of patients, all but the smallest of abscesses require drainage for resolution, regardless of the pathogen. Drainage only, without antibiotics, may suffice if the abscess is relatively isolated, with little surrounding tissue involvement. In cases of cellulitis without draining wounds or abscess, streptococci continue to be the likely etiology, and beta-lactam antibiotics are appropriate therapy, as noted in the following: In mild cases of cellulitis treated on an outpatient basis: Dicloxacillin, amoxicillin, or cephalexin, in patients who are allergic to penicillin: Clindamycin or a macrolide (clarithromycin or azithromycin), an initial dose of parenteral antibiotic with a long half-life (eg, ceftriaxone) followed by an oral agent ${ }^{(8)}$.

The aim of the current work was to evaluate the efficacy of standard treatment for cases of cellulitis and identify other treatment options if needed in our community.

\section{PATIENTS AND METHODS}

This prospective study included a total of 100 consecutive patients with non purulent cellulitis, attending at Department of General Surgery, Al-Azhar university hospitals (Al-Hussin $\&$ Said Galal hospitals). This study was conducted between $23 / 2 / 2017$ to $1 / 5 / 2018$ to allow a minimum follow-up period of at least 4 months for the last case follow upon.

\section{Ethical approval:}

The study was approved by the Ethics Board of Al-Azhar University.Prospectively enrolled patients had understood proposed investigations and treatment and signed a detailed informed consent $\mathrm{d}$, as well as, latest patient information leaflet.

For all patients, age, sex, presentation, sit of non purulent cellulitis and result were recorded. Mean age at presentation was $33.65(20-65)$ years. There were 43 men and 57 women patients.

All patients had lower limb cellulitis, and only 17 patients had an additional sit as orbital, hand and genitalia. They were included into one group consisted of one hundred patients with nonpurulent cellulitis.

Inclusion criteria: Patients with non-purulent cellulitis, localized pain, swelling, tenderness, erythema and warmth.

Exclusion criteria: Patients with purulent cellulitis.

\section{Pretreatment work up:}

All patients were subjected to history taking, clinical examination and laboratory investigations including complete blood count (CBC) and microbiological cultures (blood culture and/or aspiration).

\section{Treatment techniques}

One hundred patients with nonpurulent cellulitis were evaluated clinically, laboratory and/or radiologically. Patients were followed up at $3,5,7,15$ and 21 days to determine whether the patient was, in fact, having cellulitis according to the presence of the following clinical features: localized pain, swelling, tenderness, erythema and warmth.

Other parameters such as leucocytosis and microbiological cultures (blood culture and/or aspiration). One of standard antibiotic treatment for cellulitis (dicloxacillin, amoxicillin, and cephalexin) was started for all cases.

Cases with hypersensitivity to penicillin will be covered by clindamycin or one of the macrolides as an alternative. The clinical response will be observed at 3,5,7,15 and 21 days along with 
the proper treatment of any other coexisting comorbidity. Patients with good clinical response will continue on the same treatment, while other patient that don't show a proper clinical response will be shifted to another appropriate antibiotic according to blood culture and sensitivity or one of a wide spectra of action. The results will be recorded and analyzed statically.

\section{Statistical analysis:}

Recorded data were analyzed using the statistical package for social sciences, version 20.0 (SPSS Inc., Chicago, Illinois, USA). Quantitative data were expressed as mean \pm standard deviation (SD). Qualitative data were expressed as frequency and percentage.

\section{The following tests were done:}

- Independent-samples t-test of significance was used when comparing between two means.

- Chi-square $\left(\mathrm{x}^{2}\right)$ test of significance was used in order to compare proportions between two qualitative parameters.

- The confidence interval was set to $95 \%$ and the margin of error accepted was set to 5\%. The pvalue was considered significant as the following:

- Probability (P-value)

$$
\begin{aligned}
& \text { - P-value <0.05 was considered } \\
& \text { significant. } \\
& \text { - P-value <0.001 was considered as } \\
& \text { highly significant. } \\
& \text { - P-value >0.05 was considered } \\
& \text { insignificant. }
\end{aligned}
$$

\section{RESULTS}

\section{Demography of the patients:}

The present study was carried out on 100 patients with nonpurulent cellulitis. There were 43 males and 57 females with ratio 3:4. Mean age of patients was 33.65 years (range 20 to 65). There was significant diffrence as regards old age predominance, and male sex predominance (Table 1).

Table (1): Demography of the patients

\begin{tabular}{|l|l|}
\hline Demography & Number of cases \\
\hline Age (years) & \\
Above 45 & 65 \\
Below 45 & 35 \\
Mean & 33.65 \\
Range & $20-65$ \\
\hline Sex $(\boldsymbol{n}(\%))$ & \\
Male & $43(43 \%)$ \\
Female & $57(57 \%)$ \\
\hline
\end{tabular}

\section{Clinical features:}

There was significant difference in clinical presentation of cases as regard uncomplicated cases (mild) predominance on complicated cases (moderate to severe).

Group A: uncomplicated cases of nonpurulent cellulitis is associated with the 4 cardinal signs of infection, as follows:

- Erythema

- Pain

- Swelling

- Warmth and meet the following criteria:

- Limited area of involvement

- Minimal pain

- No systemic signs of illness (eg, fever, altered mental status, tachypnea, tachycardia, hypotension)

- No risk factors for serious illness (eg, extremes of age, general debility, immunocompromise)

Group B: complicated cases (Moderate to severe)

- Erythema

- Pain

- Swelling

- Warmth

- Cellulitis Complicating lymphedema and specific Lymphangitic spread (red lines streaking away from the area of infection)

- Malaise, chills, fever, and toxicity

- Circumferential cellulitis

- Pain disproportionate to examination findings

- Cellulitis with:

$>$ Violaceous bullae.

$>$ Cutaneous hemorrhage.

$>$ Skin sloughing.

$>$ Skin anesthesia.

$>$ Rapid progression.

$>$ Gas in the tissue.

Hypotension.

- Anatomic sites (eg, facial and especially ocular areas).

Table (2): Clinical features of the patients

\begin{tabular}{|l|l|}
\hline Clinical features & Number of cases \\
\hline Uncomplicated cases & 78 \\
\hline Complicated cases & 22 \\
\hline
\end{tabular}

There was a significant difference between cases according to treatment with (dicloxacillin, amoxicillin, and cephalexin) to the two groups as regards clinical response Table (3).

Table (3): Comparsion between good and poor clinical response within 7 days to (dicloxacillin, 
amoxicillin, and cephalexin) regarding number of cases and number according to day

\begin{tabular}{|c|c|c|}
\hline $\begin{array}{l}\text { Demography } \\
\text { of cases }\end{array}$ & $\begin{array}{l}\text { Good clinical } \\
\text { response within } \\
7 \quad \text { days } \\
\text { to(dicloxacillin, } \\
\text { amoxicillin, } \\
\text { and } \\
\text { cephalexin) }\end{array}$ & $\begin{array}{l}\text { Poor clinical } \\
\text { response within } \\
7 \quad \text { days } \\
\text { to(dicloxacillin, } \\
\text { amoxicillin, } \\
\text { and cephalexin) }\end{array}$ \\
\hline $\begin{array}{l}\text { Number of } \\
\text { cases } \\
\text { according to } \\
\text { good } \\
\text { response }\end{array}$ & 83 & 17 \\
\hline $\begin{array}{l}\text { Number } \\
\text { according } \\
\text { to days }\end{array}$ & $\begin{array}{l}43 \text { (after } 3 \text { days) } \\
26 \text { (after } 5 \text { days) } \\
14 \text { (after } 7 \text { days) }\end{array}$ & \\
\hline
\end{tabular}

Blood culture and sensitivity were done for all cases and only 2 cases had positive result with streptococcus infection and good clinical response to ampicillin sulbactam antibiotic with in 7 days Table (4).

Table (4): Comparison between positive for blood culture and sensitivity (streptococcus) and negative for blood culture sensitivity

\begin{tabular}{|l|l|l|}
\hline $\begin{array}{l}\text { Demography } \\
\text { of cases }\end{array}$ & $\begin{array}{l}\text { Positive for blood } \\
\text { culture and } \\
\text { sensitivity } \\
\text { (streptococcus) }\end{array}$ & $\begin{array}{l}\text { Negative } \\
\text { for blood } \\
\text { culture } \\
\text { sensitivity }\end{array}$ \\
\hline $\begin{array}{l}\text { Number of } \\
\text { cases } \\
\text { according to } \\
\text { good response }\end{array}$ & 2 & 98 \\
\hline
\end{tabular}

15 cases were subjected to ciprofloxacin antibiotic and 7 cases have good clinical response to -ciprofloxacin antibiotic with in 5 day and other 8 cases have good clinical response to meropenim with in 7 day's Table (5).

Table (5): Comparison between cases with good clinical response to ciprofloxacin and cases with good clinical response to meropenim

\begin{tabular}{|l|l|l|}
\hline $\begin{array}{l}\text { Demography } \\
\text { of cases }\end{array}$ & $\begin{array}{l}\text { Cases with good } \\
\text { clinical } \\
\text { response to } \\
\text { ciprofloxacin }\end{array}$ & $\begin{array}{l}\text { Cases with } \\
\text { good clinical } \\
\text { response to } \\
\text { meropenim }\end{array}$ \\
\hline $\begin{array}{l}\text { Number of } \\
\text { cases } \\
\text { according to } \\
\text { good response }\end{array}$ & 7 & 8 \\
\hline
\end{tabular}

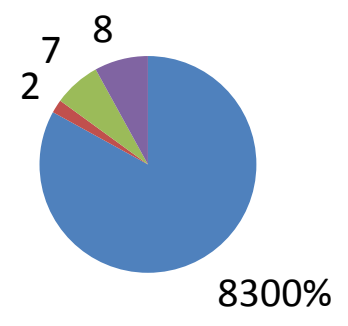

dicloxacillin, amoxicillin, and cephalexin)

sUIBACTAM

CIPRofloxacin

meropenim

Figure (1): Clinical response of cases of cellulitis to different type of antibioitic.

(83 cases have good clinical response within 7 days to (dicloxacillin, amoxicillin, and cephalexin) and 17 cases have Poor clinical response within 7 days to (dicloxacillin, amoxicillin, and cephalexin).

\section{DISCUSSION}

Certain age groups are at higher risk in some unique scenarios, such as the following:

- Historically, buccal cellulitis caused by $\mathrm{H}$ influenzae type B was more common in children younger than 3 years; vaccination against this organism may have decreased the incidence of buccal cellulitis, but recent data suggest that this source remains a consideration, even in vaccinated cohorts ${ }^{(9)}$.

- Facial cellulitis is more common in adults older than 50 years; however, pneumococcal facial cellulitis occurs primarily in young children who are at risk for pneumococcal bacteremia ${ }^{(\mathbf{1 0})}$.

- Perianal cellulitis, usually with group A beta-hemolytic Streptococcus (GABHS), occurs in children younger than 3 years ${ }^{(11)}$.

- Elderly patients with cellulitis are predisposed to thrombophlebitis. A study of an insurance database in Utah found an incidence rate of 24.6 cases per 1000 person-years. The incidence was noted to be higher in males and in those individuals aged 45-64 years ${ }^{(\mathbf{1 2})}$.

In a large epidemiologic hospital-based study on skin, soft-tissue, bone, and joint infections, $37.3 \%$ patients were identified as having cellulitis (13). Overall rates of visits increased for skin and soft-tissue infections (SSTIs) from 32.1 to 48.1 visits per 1000 
population and reached 14.2 million by 2005 , and visits for abscess and cellulitis increased from 17.3 to 32.5 visits per 1000 population and accounted for more than $95 \%$ of the increase, according to the National Ambulatory Medical Care Survey and National Hospital Ambulatory Medical Care Survey. The study provided data regarding visits by patients with SSTIs to physician offices, hospital outpatient departments, and emergency departments in the United States ${ }^{(14)}$.

Cellulitis was found to account for approximately $3 \%$ of emergency medical consultations at one United Kingdom district general hospital.

To compare the efficacy, safety, and acceptability of treatment with intravenous antibiotics for cellulitis at home and in hospital, a prospective randomised controlled trial was done at Christchurch, New Zealand. 200 patients presenting or referred to the only emergency department in Christchurch who were thought to require intravenous antibiotic treatment for cellulitis and who did not have any contraindications to home care were randomly assigned to receive treatment either at home or in hospital. Days to no advancement of cellulitis was the primary outcome measure. Days on intravenous and oral antibiotics, days in hospital or in the home care programme, complications, degree of functioning and pain, and satisfaction with site of care were also recorded. The two treatment groups did not differ significantly for the primary outcome of days to no advancement of cellulitis, with a mean of 1.50 days (SD 0.11) for the group receiving treatment at home and 1.49 days (SD $0.10)$ for the group receiving treatment in hospital (mean difference 0.01 days, 95\% confidence interval -0.3 to 0.28 ). None of the other outcome measures differed significantly except for patients' satisfaction, which was greater in patients treated at home. Treatment of cellulitis requiring intravenous antibiotics can be safely delivered at home. Patients prefer home treatment, but in this study only about one third of patients presenting at hospital for intravenous treatment of cellulitis were considered suitable for home treatment ${ }^{(15)}$.

20 children, ages 2 to 12 years, seen with cellulitis were prospectively studied. Each child had a complete blood count, a blood culture, and a culture of an aspirate from the lesion. The age of the child, the fever, and the extent of cellulitis determined whether the child was hospitalized. Outpatient antibiotic therapy was penicillin or dicloxacillin. Four children $(20 \%)$ had an infection on the upper extremity: 14 (70\%), the leg; 1 (5\%), the forehead; and one (5\%), the periorbital area. Three children (15\%) had a white blood cell (WBC) count $\geq 15,000 \mathrm{cu} \mathrm{mm}$. Two were febrile. One child with a WBC count $\leq 15,000 \mathrm{cu} \mathrm{mm}$ was febrile. Two blood cultures $(10 \%)$ were positive, both for Haemophilus influenzae. Twelve organisms were isolated from the aspirates: 8 Staphylococcus aureus (all penicillinase producing), $2 \mathrm{H}$ influenzae, 1 Streptococcus pyogenes, and 1 Pseudomonas aeruginosa. Both children with $H$ influenzae were febrile with WBC counts $\geq 15,000 \mathrm{cu} \mathrm{mm}$. Of the 17 children (85\%) treated as outpatients, all but one responded. We recommend admission and cultures of the blood and an aspirate of the lesion for all facial cellulitis and treatment with oxacillin and chloramphenicol. In a truncal or extremity cellulitis, initial therapy should be directed against $S$ aureus. If the child is febrile or the WBC count $\geq 15,000 \mathrm{cu} \mathrm{mm}, H$ influenzae is a likely pathogen and thus intravenous chloramphenicol must be used after cultures are obtained $^{(\mathbf{1 6})}$.

30 adult patients with cellulitis were prospectively studied including many who were hospitalized with significant underlying medical problems. Needle aspiration of both central and leading edge areas of their lesions was performed in an attempt to establish a bacteriologic diagnosis. Potential pathogens were isolated by this technique in only $10 \%$ of the patients. Neither site of aspiration was clearly superior in terms of culture yield. Because aspirate Gram stains and cultures so rarely provided useful bacteriologic information, they were seldom helpful in guiding antibiotic selection or in influencing the outcome of treatment in most patients. However, clinical information, as well as results of primary lesion cultures when obtainable, may be used to successfully select therapy in most cases of adult cellulitis. On the basis of our results, needle aspiration may not be justified as a routine diagnostic procedure for all adults with cellulitis, though it may still be useful in selected patients ${ }^{(17)}$.

To assess the cost-effectiveness of blood cultures for patients with cellulitis, a retrospective review was conducted of clinical and microbiological data for all 757 patients admitted to a medical center because of communityacquired cellulitis during a 41-month period. Blood cultures were performed for 553 patients (73\%); there were a total of 710 blood samples (i.e., a mean of 1.3 cultures were performed per patient). In only 11 cases $(2.0 \%)$ was a significant patientspecific microbial strain isolated, mainly $\beta$ hemolytic streptococci (8 patients $(73 \%))$. An 
organism that was considered a contaminant was isolated from an additional 20 culture bottles (3.6\%). The cost of laboratory workup of the 710 culture sets was $\$ 36,050$. Isolation of streptococci led to a change from empirical treatment with cefazolin to penicillin therapy for 8 patients. All patients recovered. In conclusion, the yield of blood cultures is very low, has a marginal impact on clinical management, and does not appear to be cost-effective for most patients with cellulitis ${ }^{(\mathbf{1 8})}$.

In this study we tried to look at another treatment of cellulitis, there was significant difference as regards old age predominance, and female sex predominance. There were 43 males and 57 females with male to female ratio 3:4. Mean age of our patients was 33.65 years (range 20 to 65 years).

There was significant difference in clinical presentation of cases as regard uncomplicated cases (mild) predominance on complicated cases (moderate to severe). 78 cases complicated and 22 cases noncomplicated.

The all patients had lower limb cellulitis, and only 17 patients had an additional sit as 7 cases at the orbit, 6 cases at hand and 4 cases genitalia.

Only 4 cases have hypersensitivity to penicillin but 96 cases have normal sensitivity to penicillin.

There was a significant difference between cases according to treatment with (dicloxacillin, amoxicillin, and cephalexin) to the two groups as regards clinical response.

83 cases have good clinical response within 7 days to (dicloxacillin, amoxicillin, and cephalexin) and 17 cases have Poor clinical response within 7 days to (dicloxacillin, amoxicillin, and cephalexin).

All cases were subjected to blood culture and sensitivity and only 2 cases have positive result with streptococcus infection and good clinical response to ampicillin sulbactam antibiotic within 7 day. 7 cases have good clinical response to ciprofloxacin antibiotic within 5 day and 8 cases have good clinical response to meropenim within 7 days.

\section{CONCLUSION}

Dicloxacillin, amoxicillin, or cephalexin are effective in most cases of cellulitis with gram positive organisms as streptococcal and staphylococcal species. At more serious or sever nontraditional cellulitis organisms, including gram-negative rods (e.g. Pseudomonas, Proteus, Serratia, Enterobacter, Citrobacter), anaerobes, and others (eg, Helicobacter cinaedi, Fusarium species) and fungi (eg, Cryptococcus) Fluoroquinolones are more effective alone or with combination with (Dicloxacillin, amoxicillin, or cephalexin) or clindamycin or antifungal.

\section{REFERENCES}

1. Busch BA, Ahern MT, Topinka $M$ et al. (2010): Eschar with cellulitis as a clinical predictor in community-acquired MRSA skin abscess. J Emerg Med., 38(5):563-6.

2. Brodell LA, James DB, Robert TB (2014): Recurrent lymphangitic cellulitis syndrome: A quintessential example of an immunocompromised district. Clinics in Dermatology, 32: 621-62.

3. Stevens DL, Bisno AL, Chambers HF et al. (2014): Practice guidelines for the diagnosis and management of skin and soft tissue infections: 2014 update by the Infectious Diseases Society of America. Clin Infect Dis., 59(2):147-59.

4. Joseph J, Abraham S, Soman A et al. (2010): Cellulitis: a bacterial skin infection, their causes, diagnosis and treatment. WJPPS., 3: 308-326.

5. Woo PC, Lum PN, Wong SS et al. (2000): Cellulitis complicating lymphoedema. Eur $\mathbf{J}$ Clin Microbiol Infect Dis., 19(4):294-7.

6. Swartz MN (2004): Clinical practice. Cellulitis. N Engl J Med., 350(9):904-12.

7. Tayal VS, Hasan N, Norton HJ et al. (2006): The effect of soft-tissue ultrasound on the management of cellulitis in the emergency department. Acad Emerg Med., 13(4):384-8.

8. Falagas ME, Karagiannis AK, Nakouti T et al. (2010): Patients included in randomised controlled trials do not represent those seen in clinical practice: focus on antimicrobial agents. International Journal of Antimicrobial Agents, 36: 1-13.

9. Cieslak TJ, Rajnik M, Roscelli JD (2008): Immunization against Haemophilus influenzae type $B$ fails to prevent orbital and facial cellulitis: results of a 25-year study among military children. Mil Med., 173(10):941-4.

10. Givner LB, Mason EO, Barson WJ et al. (2000): Pneumococcal facial cellulitis in children. Pediatrics, 106(5):61-5.

11. Kokx NP, Comstock JA, Facklam RR (1987): Streptococcal perianal disease in children. Pediatrics, 80(5):659-63.

12. Ellis Simonsen SM, van Orman ER, Hatch BE et al. (2006): Cellulitis incidence in a defined population. Epidemiol Infect., 134(2):293-9. 
13. Lipsky BA, Weigelt JA, Gupta $\mathrm{V}$ et al. (2007): Skin, soft tissue, bone, and joint infections in hospitalized patients: epidemiology and microbiological, clinical, and economic outcomes. Infect Control Hosp Epidemiol., 28(11):1290-8.

14. Hersh AL, Chambers HF, Maselli JH et al. (2008): National trends in ambulatory visits and antibiotic prescribing for skin and softtissue infections. Arch Intern Med., 168(14):1585-91.
15. Corwin P, Toop L, McGeoch G et al. (2005): Randomised controlled trial of intravenous antibiotic treatment for cellulitis at home compared with hospital. BMJ., 330(7483):12933.

16. Fleisher G, Ludwig S (1980): Cellulitis: A prospective study. Annals of Emergency Medicine, 9(5): 246-249.

17. Newell PM, Norden CW (1988): Value of needle aspiration in bacteriologic diagnosis of cellulitis in adults. Journal of Clinical Microbiology, 26(3):401-4.

18. Perl B, Gottehrer NP, Raveh D et al. (1999): Cost-effectiveness of blood cultures for adult patients with cellulitis. Clinical Infectious Diseases, 29(6):1483-8. 